\title{
Social networks: evolving graphs with memory dependent edges
}

\section{Article}

Accepted Version

this version from preprint series

Grindrod, P. and Parsons, M. (2011) Social networks: evolving graphs with memory dependent edges. Physica A, 390 (2. pp. 3970-3981. doi: https://doi.org/10.1016/j.physa.2011.06.015 Available at https://centaur.reading.ac.uk/26936/

It is advisable to refer to the publisher's version if you intend to cite from the work. See Guidance on citing.

To link to this article DOI: http://dx.doi.org/10.1016/j.physa.2011.06.015

All outputs in CentAUR are protected by Intellectual Property Rights law, including copyright law. Copyright and IPR is retained by the creators or other copyright holders. Terms and conditions for use of this material are defined in the End User Agreement.

\section{www.reading.ac.uk/centaur}

\section{CentAUR}

Central Archive at the University of Reading

Reading's research outputs online 


\title{
Department of Mathematics
}

\author{
Preprint MPS_2010-02
}

4 March 2010

\section{Social Networks: Evolving Graphs with Memory Dependent Edges}

by

Peter Grindrod and Mark Parsons

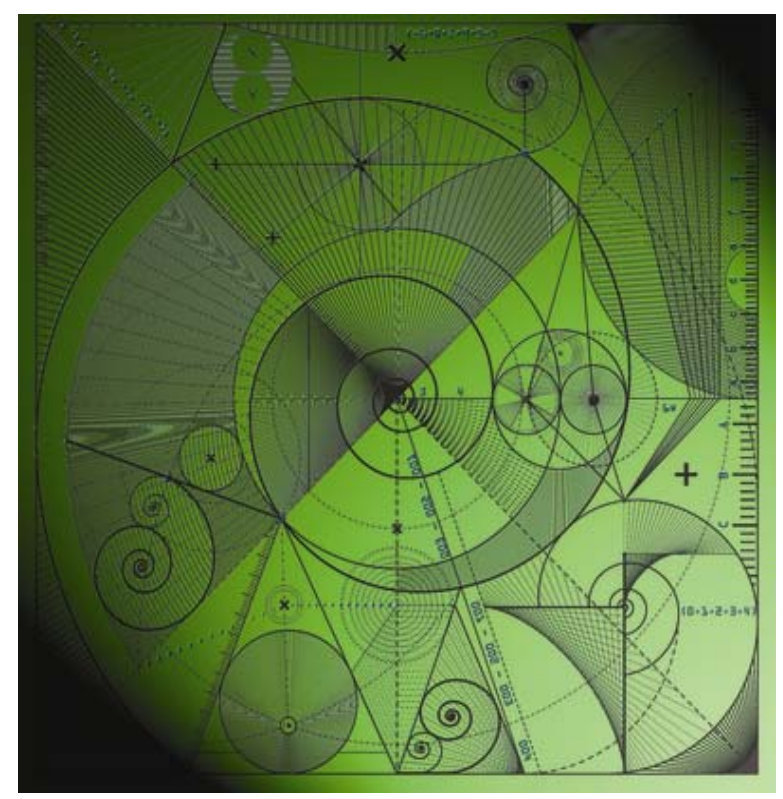




\title{
Social Networks: Evolving Graphs with Memory Dependent Edges
}

\author{
Peter Grindrod Mark Parsons
}

February 28, 2010

\begin{abstract}
The plethora, and mass take up, of digital communication technologies has resulted in a wealth of interest in social network data collection and analysis in recent years. Within many such networks the interactions are transient: thus those networks evolve over time. In this paper we introduce a class of models for such networks using evolving graphs with memory dependent edges, which may appear and disappear according to their recent history. We consider time discrete and time continuous variants of the model. We consider the long term asymptotic behaviour as a function of parameters controlling the memory dependence. In particular we show that such networks may continue evolving forever, or else may quench and become static (containing immortal and/or extinct edges). This depends on the existence or otherwise of certain infinite products and series involving age dependent model parameters. To test these ideas we show how model parameters may be calibrated based on limited samples of time dependent data, and we apply these concepts to three real networks: summary data on mobile phone use from a developing region; online social-business network data from China; and disaggregated mobile phone communications data from a reality mining experiment in the US. In each case we show that there is evidence for memory dependent dynamics, such as that embodied within the class of models proposed here.
\end{abstract}




\section{Introduction}

An evolving graph is a sequence of graphs, $\left\{G_{i} \mid i=\ldots, 0,1,2, \ldots\right\}$, defined on a finite set of vertices, satisfying a given stochastic evolution process:

$$
P\left(G_{i+1} \mid G_{i}, G_{i-1}, \ldots\right) .
$$

Here $G_{i}$ should be thought of as being the state of the evolving graph at the $i$ th discrete time step. We do not allow for the successive addition of vertices at every time step, as in growth or attachment models.

These graphs have been motivated in recent work, in $[14,3]$ for example, and models of evolving graphs have been discussed by Grindrod and Higham [8], under the assumption that the evolution obeys a single step Markov process: $P\left(G_{i+1} \mid G_{i}\right)$. Instead, here we wish our network to be dependent upon longer segments from the entire history of the graph. Examples of such systems exist across a variety of social networks, ranging from communicative $[6,10]$ and transportation [12] issues, to the "Reality Mining" project [4], for which our modelling approach is relevant and offers an additional perspective into their behaviour.

In fact we shall consider a subclass of evolving graphs with memory, where each edge is independent and depends on its own history. These are evolving graphs containing memory dependent edges.

In section 2 we define our terminology and then examine four possible alternatives for the long term behaviour. We shall derive a means by which to determine into which category a network will fall given its defining parameters, in the process introducing the concepts of immortal and extinct edges, and identifying both static (quenched) and dynamic steady states. In section 3 we consider graphs evolving continuously in time, rather than discrete time steps.

We are interested in applications to communicative and social networks (enabled by a variety of digital technologies). In such cases the vertices usually represent individual users (people) while the edges (directed or not) are a form of timed interactions. Section 4 offers an example, where we observe a dynamically evolving equilibrium. Next we consider the inverse problem for such systems: given some data from a graph assumed to be of this type, make an estimate for the associated model parameters and thus infer its long 
term behaviour. We illustrate the relevance of memory dependent edges in the life cycle data extracted from the Reality Mining experiments [4].

\section{Edge Birth and Death Rates}

We consider graphs made up of independent edges, where the evolution of each edge depends upon its own history, and in particular upon how long an edge has, or has not, been present.

\subsection{Network Properties}

Let $e$ denote a possible edge connecting a pair of distinct vertices which is dynamically evolving with time. At any time step, if $e$ is present then we will say it is alive, and similarly if $e$ is absent then we will say it is dead. The evolution of the network will be governed by two quantities called the edge birth and death rates, which determine the probability that an edge will die or be born at the next time step:

The birth rate for $e$ is denoted by $\alpha(k)$, where $k$ is the number of consecutive time steps for which $e$ has been absent, up to and including the current time step.

The death rate for $e$ is denoted by $\omega(k)$, where $k$ is the number of consecutive time steps for which $e$ has been present, up to and including the current time step.

Let $p_{m, k}$ denote the probability that $e$ is present at time step $m$, and has been present for exactly $k$ consecutive time steps, up to and including time step $m$. Similarly let $q_{m, k}$ denote the probability that $e$ is absent at time step $m$, and has been absent for exactly $k$ consecutive time steps, up to and including $m$.

Then it follows that

$$
p_{m+1, k+1}=p_{m, k}(1-\omega(k)), k=1,2, \ldots,
$$

and similarly

$$
q_{m+1, k+1}=q_{m, k}(1-\alpha(k)), k=1,2, \ldots
$$


Total births and deaths imply

$$
\begin{aligned}
p_{m+1,1} & =\sum_{k=1}^{\infty} q_{m, k} \alpha(k) \\
q_{m+1,1} & =\sum_{k=1}^{\infty} p_{m, k} \omega(k) .
\end{aligned}
$$

Equations (1-4) define our evolving edge model.

Now consider the long-term behaviour of this process. Suppose that a dynamic steady state occurs, with an equilibrium between the probability of $e$ being newly created and lost during the transition between successive time steps. Then $p_{m, k}$ and $q_{m, k}$ will be independent of $m$, say, $p_{m, k}=p_{k}^{*}$ and $q_{m, k}$ $=q_{k}^{*}$ for $k=1,2, \ldots$. We will have

$$
p_{1}^{*}=q_{1}^{*}=\gamma
$$

where $\gamma$ is the probability that $e$ will be born at each time step, and is also the probability that $e$ will be die at each time step.

It follows from (1) and (2), that

$$
p_{k}^{*}=\gamma \prod_{j=1}^{k-1}(1-\omega(j))
$$

and

$$
q_{k}^{*}=\gamma \prod_{j=1}^{k-1}(1-\alpha(j))
$$

However

$$
\sum_{1}^{\infty} p_{k}^{*}+\sum_{1}^{\infty} q_{k}^{*}=1
$$

and so

$$
\gamma=\frac{1}{\Omega+A}
$$

provided that

$\Omega=1+(1-\omega(1))+(1-\omega(1))(1-\omega(2))+(1-\omega(1))(1-\omega(2))(1-\omega(3))+\ldots$ 
and

$A=1+(1-\alpha(1))+(1-\alpha(1))(1-\alpha(2))+(1-\alpha(1))(1-\alpha(2))(1-\alpha(3))+\ldots$

are both finite.

Conversely if the sums $\Omega$ and $A$ exist then $\gamma$ in (8) is positive, and so $p_{k}^{*}$ and $q_{k}^{*}$ given by (6) and (7) are equilibrium solutions of the edge dynamics (1-4).

If $\alpha$ and $\omega$ are constants (independent of $k$ ) we have the Markov model given in [8]: in that case a dynamic equilibrium obviously exists.

In general the existence of $\Omega$ and $A$ depends on the chosen birth and death rates, so next we consider a case where the longer edges manage to survive, the more resilient they become (and the longer edges are absent the less likely they are to be born). Let us assume the power law forms

$$
\omega(k)=\frac{b}{k^{r}}, \alpha(k)=\frac{b^{\prime}}{k^{r^{\prime}}},
$$

where $b$ and $b^{\prime} \in(0,1)$ are fixed, and the parameters $r \geq 0$ and $r^{\prime} \geq 0$ govern the sensitivity of the rates to $k$. Notice that any results obtained for the existence of $\Omega$ will have an equivalence for $A$.

Thus for $\omega(k)$ given by (9), $\Omega$ converges for $r<1$, and diverges otherwise. This follows directly from Raabe's test [16].

\subsection{Immortal and Extinct Edges}

The probability that any edge, which has been present for precisely $z=$ $1,2, \ldots$ time steps, remains forever, and becomes "immortal", is equal to the probability that it never again dies and we write:

$$
P_{i m m}(z)=\prod_{k=z}^{\infty}(1-\omega(k)) .
$$

Let us denote $P_{i m m}(1)=P_{i m m}$, so that

$$
P_{i m m}(z)=\frac{P_{i m m}}{\prod_{k=1}^{z-1}(1-\omega(k))} .
$$


It is known [13] that if $0<s_{k}<1$ for all $k$, then $\prod_{k=1}^{\infty}\left(1-s_{k}\right)>0$ if and only if $\sum_{k=1}^{\infty} s_{k}<\infty$. Hence we have

$$
P_{i m m}=\prod_{k=1}^{\infty}(1-\omega(k))>0 \Leftrightarrow r>1,
$$

and therefore $P_{\text {imm }}(z)>0$ for all $z=1,2, \ldots \Leftrightarrow r>1$.

Analogously we define the probability that an edge which has been absent for precisely $z=1,2, \ldots$ time steps, will remain so for ever and becomes "extinct" via

$$
P_{\text {ext }}(z)=\prod_{k=z}^{\infty}(1-\alpha(k))
$$

and $P_{\text {ext }}=P_{\text {ext }}(1)$.

Given $\alpha(k)$ and $\omega(k)$ it remains to calculate the infinite products $P_{i m m}$ and $P_{\text {ext }}$. For some cases given by (9) this may be resolved by the Euler-Prudnikov identity, which says that for $\beta>0$, and $r=2,3, \ldots[1]$

$$
\beta^{r} \prod_{k=1}^{\infty}\left(1-\frac{\beta^{r}}{k^{r}}\right)=-\prod_{j=0}^{r-1} \frac{1}{\Gamma\left(-\beta e^{2 \pi j i / r}\right)} .
$$

Hence taking $\beta=b^{(1 / r)}$ we obtain

$$
P_{i m m}=-\frac{1}{b} \prod_{j=0}^{r-1} \frac{1}{\Gamma\left(-b^{1 / r} e^{2 \pi j i / r}\right)}
$$

(and similarly for $P_{\text {ext }}$ ). This is useful as we can calculate $P_{i m m}$ exactly using this finite product.

\subsection{Long Term Behaviour}

Now since both $A$ and $\Omega$ may individually converge or diverge, in determining the system's long term behaviour we have four different scenarios to consider. Suppose for simplicity that we have a graph made up of edges, each obeying similar stochastic dynamics. 


\section{$A$ and $\Omega$ Both Converge}

In this case, as we have seen, $P_{i m m}(z)=P_{e x t}=0$ and

$$
\gamma=\frac{1}{\Omega+A}>0
$$

exists. The expected long-term structure of a network consisting of such (similar) edges is that of an Erdos-Renyi random graph, since at any long term time step each edge has a probability of $\Omega /(\Omega+A)$ of being present. The central limit theorem tells us that for a large number of vertices, $N$, the degree distribution must necessarily be Gaussian (with a modal value of $\left.\frac{(N-1) \Omega}{\Omega+A}\right)$.

\section{$A$ Converges and $\Omega$ Diverges}

In this case, $P_{i m m}>0$ and $P_{e x t}=0$. So each edge will eventually become immortal, and hence the long term steady state will be a static complete graph (clique).

\section{$A$ Diverges $\Omega$ Converges}

In this case, $P_{i m m}=0$ and $P_{e x t}>0$. So each edge will eventually become extinct, and hence the long term steady state will be a static empty graph.

\section{$A$ and $\Omega$ Both Diverge}

In this case both $P_{i m m}, P_{e x t}>0$. So each edge will eventually become either immortal or extinct and there is no dynamic equilibrium. Thus any possible static (quenched) graph, with each edge either immortal or extinct, is a valid steady state. In the appendix we show how to determine the probability that any edge (present or absent) becomes immortal or extinct depending upon its recent history. 


\section{Time continuous models}

So far we have considered graphs with edges that evolve over discrete time steps. Here we pause to consider the continuous time case. Just as time, $t$, become continuous so must the age, $s$, (in units of time) of a present or absent edge. Let $p(t, s)$ denote the probability density for the edge being present at time $t$ and having been so for a time of $s>0$. Let $q(t, s)$ denote the probability density for the edge being absent at time $t$, and having been so for a time of $s>0$. Let $\alpha(s)$ and $\omega(s)$ denote the rate at which edges appear or disappear, as a function of the age, $s$, of the continuous absence or presence of the edge, respectively.

Then conservation of mass implies (using subscript notation for partial derivatives)

$$
\begin{aligned}
& p_{t}(t, s)+p_{s}(t, s)=-\omega p(t, s), \quad s>0 ; \quad p(t, 0)=\int_{0}^{\infty} \alpha(s) q(t, s) d s ; \\
& q_{t}(t, s)+q_{s}(t, s)=-\alpha q(t, s), \quad s>0 ; \quad q(t, 0)=\int_{0}^{\infty} \omega(s) p(t, s) d s .
\end{aligned}
$$

Note also that, for all $t$, we have $1=\int_{0}^{\infty} p(t, s)+q(t, s) d s$.

These equations have solutions (where they exist) given by

$$
p(t, s)=P_{0}(t-s) e^{-\int_{0}^{s} \omega\left(s^{\prime}\right) d s^{\prime}}, \quad p(t, s)=Q_{0}(t-s) e^{-\int_{0}^{s} \alpha\left(s^{\prime}\right) d s^{\prime}},
$$

where the rate of births $P_{0}(t)(=p(t, 0))$ and deaths $Q_{0}(t)(=q(t, 0))$ must satisfy

$$
P_{0}(t)=\int_{0}^{\infty} \alpha(s) Q_{0}(t-s) e^{-\int_{0}^{s} \alpha\left(s^{\prime}\right) d s^{\prime}} d s
$$

and

$$
Q_{0}(t)=\int_{0}^{\infty} \omega(s) P_{0}(t-s) e^{-\int_{0}^{s} \omega\left(s^{\prime}\right) d s^{\prime}} d s
$$

Suppose this approaches a dynamic equilibrium for large $t$, where both $P_{0}$ and $Q_{0}$ tend to $\gamma>0$, say. Then we must have

$$
1=\gamma\left\{\int_{0}^{\infty} e^{-\int_{0}^{s} \omega\left(s^{\prime}\right) d s^{\prime}} d s+\int_{0}^{\infty} e^{-\int_{0}^{s} \alpha\left(s^{\prime}\right) d s^{\prime}} d s\right\}
$$


If either integral does not exist then no such dynamical steady state exists and it is clear that the evolving graph will quench, just at it did for the time discrete case. However, for $\omega\left(s^{\prime}\right)=\frac{b}{s^{\prime r}}$ as in the discrete case, $\int_{0}^{\infty} e^{-\int_{0}^{s} \omega\left(s^{\prime}\right) d s^{\prime}}$ exists only for $0<r<1$, and similarly for $\alpha\left(s^{\prime}\right)=\frac{b^{\prime}}{s^{\prime r^{\prime}}}$. Hence $\gamma$ exists, and we obtain a dynamic steady state, only when $r, r^{\prime}<1$, and we obtain a static steady state otherwise, as previously shown.

Hence the time continuous case is similar to that described for the time discrete case, with the asymptotic summability of the birth and death rates determining the long term behaviour. So for independent memory dependent edge dynamics there is no loss of alternative solution structure in passing from the continuous time to the discrete time formulations.

\section{Telecommunication Example}

This example uses 65000 mobile telephone users from a developing nation where almost all phone use is via mobiles, and each individual's weekly received number of distinct counterparties is documented for nine consecutive weeks (the calls sent counterparties are very similar in distribution [6]). Since no information was provided stating when these customers registered with this specific provider, the first two weeks of data has been discarded, since individuals' behaviour regarding telephone usage might be atypical in the immediate period after new registration.

Each graph in Figure 1 shows the joint distribution of the received counterparties by each individual on successive weeks, which we shall denote $W_{k}{ }_{k+1}$. If we had obtained a static steady state, we would necessarily only observe entries on the principle diagonal, however Figure 1 clearly illustrates dispersion occurring, highlighting that our system may be in a dynamic state $(\gamma>0)$.

It is possible that, since our system is dynamic, we have yet to achieve steady state, however the sequence of graphs in Figure 1 do not appear to be converging towards the diagonal over time, as further shown by Table 1 (otherwise we would expect entries comparing graphs chronologically far apart, say $W_{34}$ and $W_{89}$, to be substantially less that 1 ). In Figure 2 , we show the distributions of the number of distinct counterparties received for each 


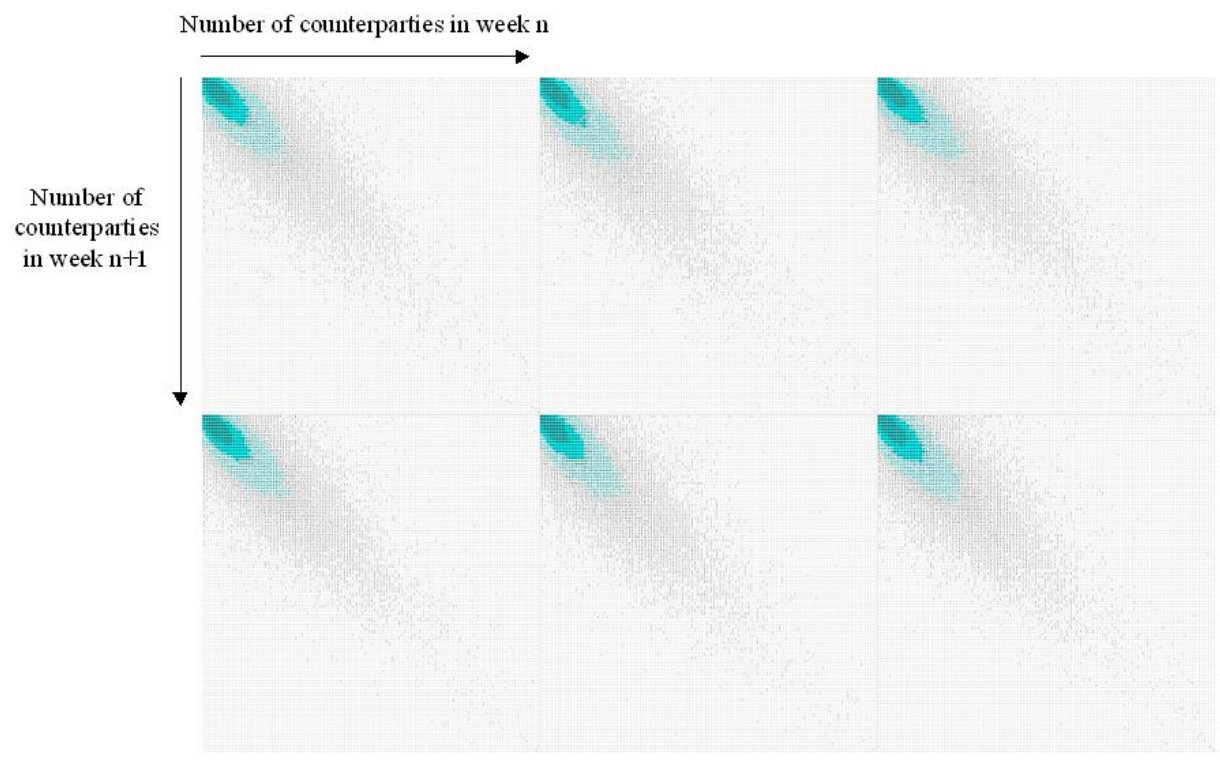

Figure 1: A sequence of 6 pivot tables showing the joint distribution of individuals' number of distinct counterparties in successive weeks, starting from a comparison of weeks 3 and 4, up to a comparison of weeks 8 and 9. A white cell indicates a value $\leq 25$, light blue indicates a value in the range 26-125, blue indicates a value in the range 126-300, and a dark blue cell indicates a value $>300$. 


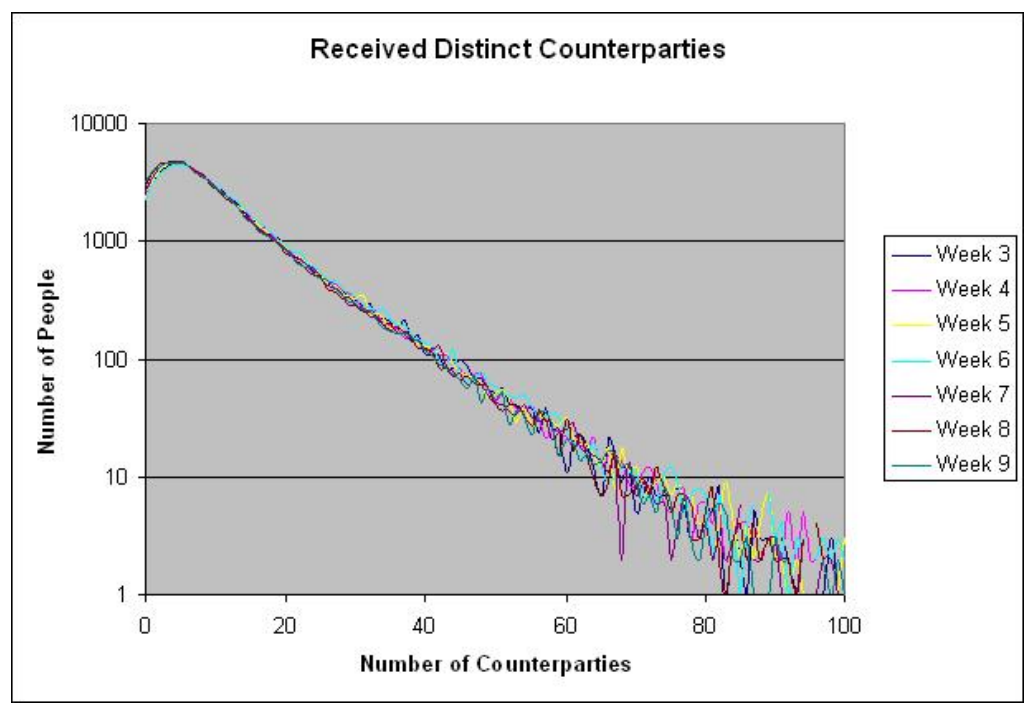

Figure 2: A scatter plot illustrating the number of people with specific number of counterparties, for weeks 3 through 9 .

week (the weekly degree distribution), 3 through 9 . These exhibit only very minor discrepancies, so the distribution of users with respect to their number of counterparties remains relatively constant from week on week, whereas the identities of these people is changing (Figure 1). In the vocabulary here, it seems that our network has largely achieved a dynamic steady state.

\begin{tabular}{l|c|c|c|c|c|c} 
& $W_{34}$ & $W_{45}$ & $W_{56}$ & $W_{67}$ & $W_{78}$ & $W_{89}$ \\
\hline$W_{34}$ & 1.0000 & & & & & \\
\hline$W_{45}$ & 0.9963 & 1.0000 & & & & \\
\hline$W_{56}$ & 0.9950 & 0.9950 & 1.0000 & & & \\
\hline$W_{67}$ & 0.9922 & 0.9913 & 0.9866 & 1.0000 & & \\
\hline$W_{78}$ & 0.9933 & 0.9922 & 0.9892 & 0.9925 & 1.0000 & \\
\hline$W_{89}$ & 0.9889 & 0.9891 & 0.9873 & 0.9857 & 0.9936 & 1.0000
\end{tabular}

Table 1: This shows the normalised dot products between the matrices $W_{34}$ through to $W_{89}$, from Figure 1, to measure their respective similarities to each other.

However, if all possible edges (counterparties) occurred independently, then we would necessarily obtain a Poisson distribution in Figure 2. This is not the case since the variance is too high. Instead the graph section up to the 
modal value each week approximately follow a Poisson distribution, but the distribution of the graph's tail more accurately matches that of a BarabasiAlbert [5], or a Pareto [17] distribution. One explanation for this could be that individuals' behaviours are linked to their number of weekly distinct counterparties. For example there may be two or more populations here, say personal users, business users, etc., having fewer and many counterparties respectively. Hence the long tail may correspond to the evolution of the business users (high degree vertices) with longer expected value and variance.

\section{Model Calibration}

Here we suppose we have sequential data from an edge having a birth and death function $\alpha(k)$ and $\omega(k)$ given by (9). Since we may not have reached any of the possible long term equilibria, we may wish to know how close a system is to boundary between static (quenching) and dynamic steady states (how close $r$ or $r^{\prime}$ is to 1 ).

We may use Bayes' formula to estimate our parameters. Here we focus on the death rate parameters. Given the data in the form of the observed sequential evolution of edges, we wish to find values for $r$ and $b$ in (9) that maximise $P((b, r) \mid D a t a)$. For our case we shall use an unnormalised Bayes' posterior distribution:

$$
P((b, r) \mid \text { Data })=P(\text { Data } \mid(b, r)) \cdot P_{\text {Prior }}(b, r)
$$

Further, let us take $P_{\text {Prior }}(b, r)$ to be improper, and equal to one for all $0<b<1$ and $r>0$, and zero otherwise. So $P((b, r) \mid$ Data $)=P($ Data $\mid(b, r))$.

Notice that the probability an edge dies after existing for precisely $k$ time steps is:

$$
\left(\prod_{j=1}^{k-1}(1-\omega(j))\right) \cdot \omega(k)
$$

Let 'life cycle' refer to the period from an observed edge's (re)birth, until its death and let $n(k)=$ the number of life cycles of length $k$ in our data (each life cycle is independent in this model). We can then see that the probability 
that $n(k)$ edges die after existing for precisely $k$ time steps, is:

$$
\left(\left(\prod_{j=1}^{k-1}(1-\omega(j))\right) \cdot \omega(k)\right)^{n(k)}
$$

and hence the probability of observing our data is:

$$
P(\operatorname{Data} \mid(b, r))=\prod_{k=1}^{K}\left(\left(\prod_{j=1}^{k-1}(1-\omega(j))\right) \cdot \omega(k)\right)^{n(k)}
$$

where $K$ is the length of the largest observed life cycle. Substituting this into equation (10), and taking logs to ease the calculations, we see that:

$$
\ln (P((b, r) \mid \text { Data }))=\sum_{k=1}^{K} n(k)\left(\sum_{j=1}^{k-1} \ln (1-\omega(j))+\ln \omega(k)\right)
$$

and so we can estimate the parameters $r$ and $b$ by maximising (11).

In order to test the method we shall apply it to artificial test data created with known parameters $b=0.3, r=0.7, K=195$, and a total of 500 life cycles. This method estimates modal values $b \approx 0.2701$ and $r \approx 0.6013$. The contour plot (Figure 3) then summarises the posterior distribution.

Next we apply this calibration technique to the "Reality Mining" project data [4], which contains telecommunicative exchanges between 106 different members of faculty ( $90 \%$ graduate students, $10 \%$ staff), from a major university, over 48 consecutive weekly periods (shown in Figure $4^{1}$ ).

To confirm our premise that such data is memory dependent we must approximate our parameter $r$ by calculating the lengths of the life cycles (using weeks as our units) for each edge formed within our 48 week period (6114 in total). Applying (11) to this data then allows us to construct a contour plot (Figure 5) and yields our parameter estimations of $b \approx 0.63$ and $r \approx 0.51$. Notice that since $r$ is not close to 0 , our premise of memory dependence of $\omega(k)$ is confirmed.

\footnotetext{
${ }^{1}$ The increase and decrease in activity can be explained by the start and end of the academic year, respectively.
} 


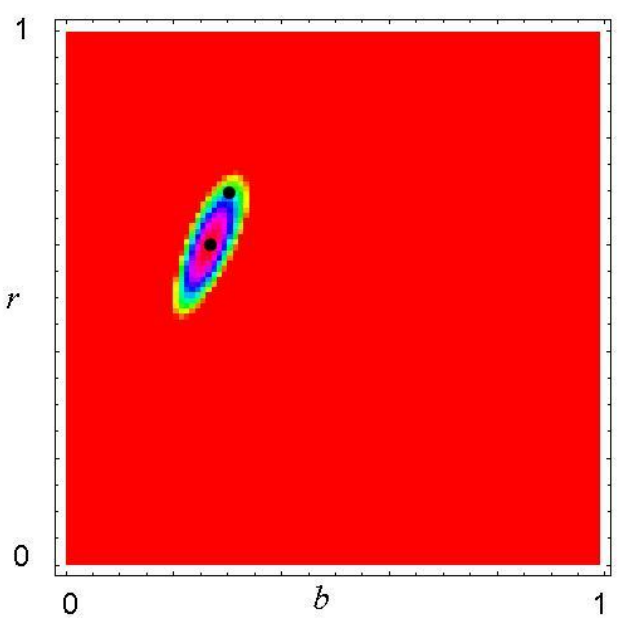

Figure 3: A contour plot of our Bayesian estimates for $r$ and $b$ from our artificial data, with the actual values for $r$ and $b$ (0.7 and 0.3 respectively) highlighted.

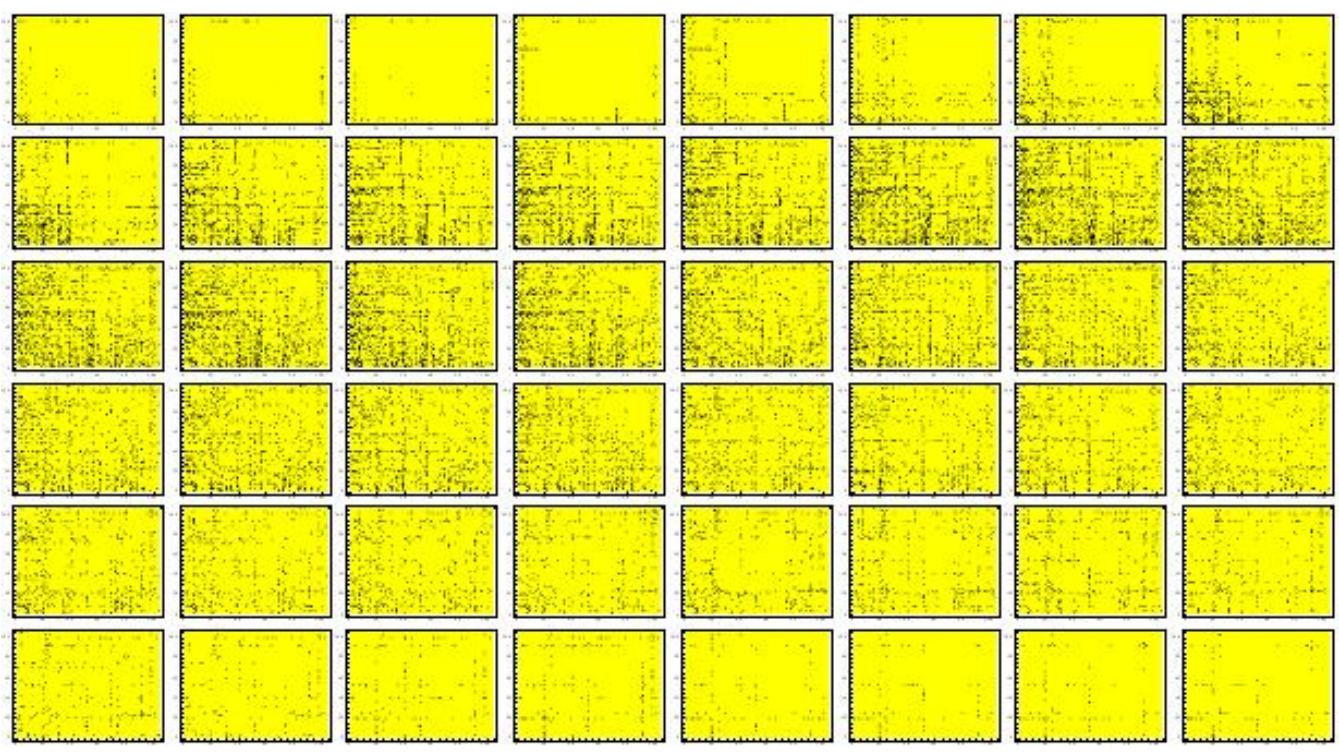

Figure 4: A series of 48 consecutive weekly adjacency matrices, indicating when communication did (black cell) and did not (yellow cell) take place between individuals each week, [4]. 

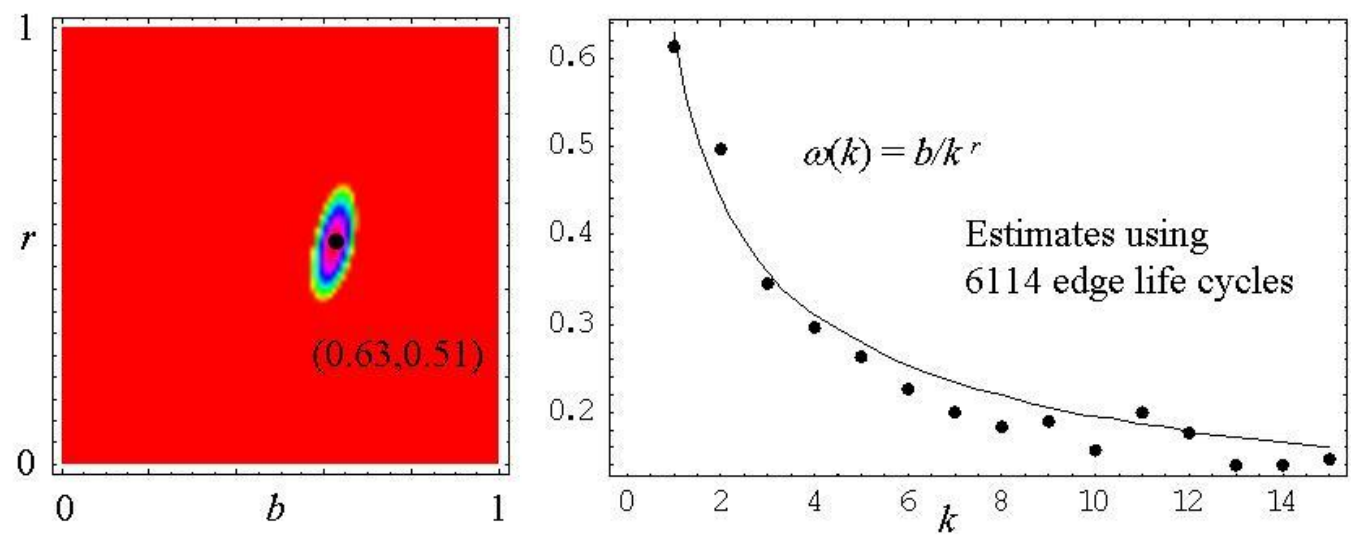

Figure 5: A contour plot of the Bayesian estimates for $r$ and $b$ from the Reality Mining data [4], together with a plot of the resulting approximated $\omega(k)$ function using these parameters, against the actual life cycle data.

Now let us also consider the Chinese professional social network data analysed in [10], and examine the behaviour of the weekly birth rate for "links" (edges). This network is very large and examines a total of 273209 pairwise links (edges) formed between 223482 registered members (vertices), over about 100 weeks.

First, for each member we defined the joining time to be the (absolute) time at which the member made his first link, and thus joined the network. Figure 6 shows the full network adjacency matrix with members arranged in the order in which they joined the network (notice that, in this data set, once an edge is formed it can never die).

We analysed each link in turn, and calculated its "time to birth", given by the difference between the absolute time of the link's formation and the maximum of the joining times of the connecting parties. This distribution is shown in Figure 7 below. Notice that the majority of the links (83\%) are formed as one of the pair joins the network (meaning they are formed at the joining time of one or the other connecting parties).

If we focus on those links made organically, that is not at joining times, but as a result of networking between pre-existing members, we see a familiar pattern: the longer a link remains unmade the less likely it is to be born 


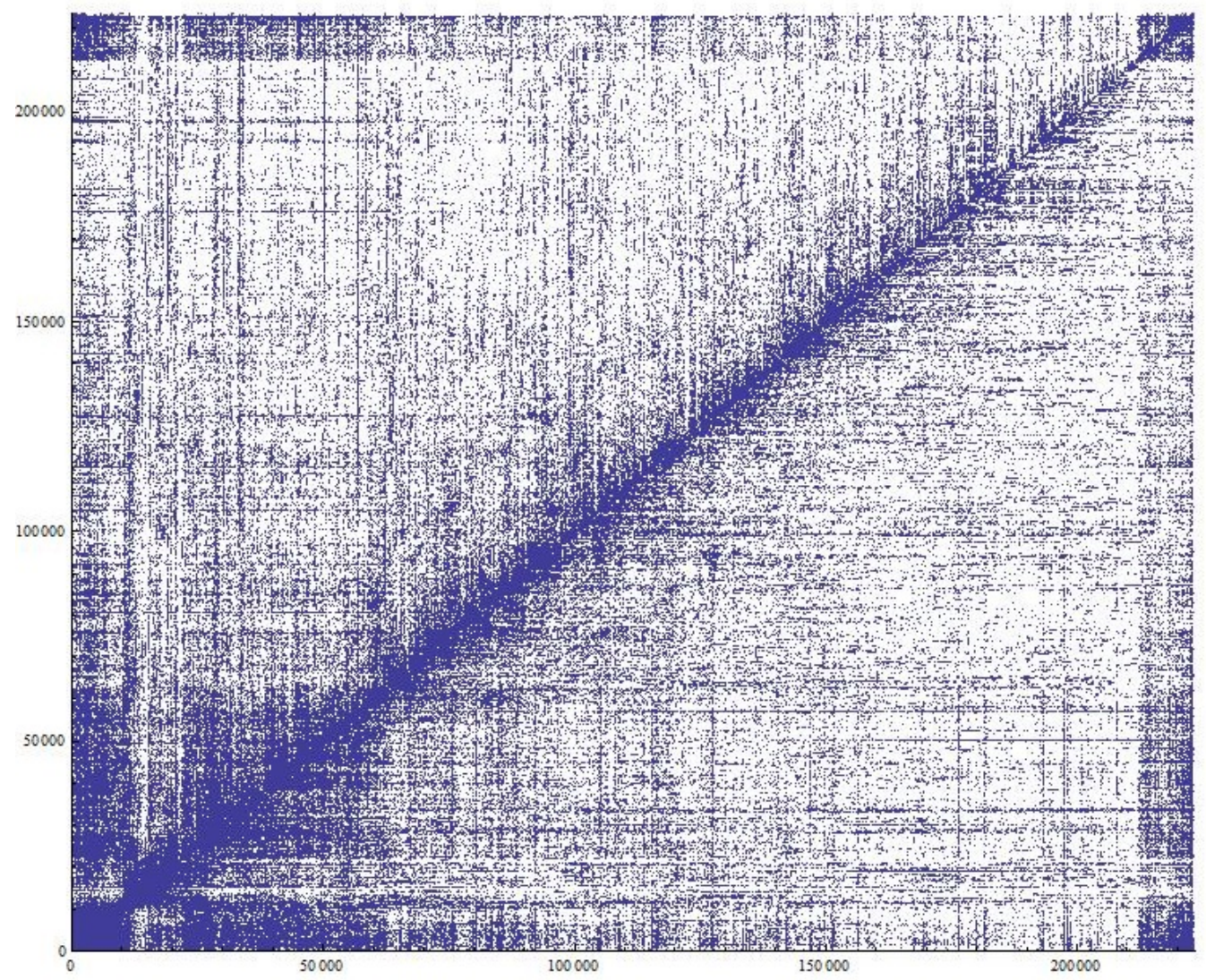

Figure 6: An adjacency matrix showing the full social network of 223482 individuals using all the data from [10].

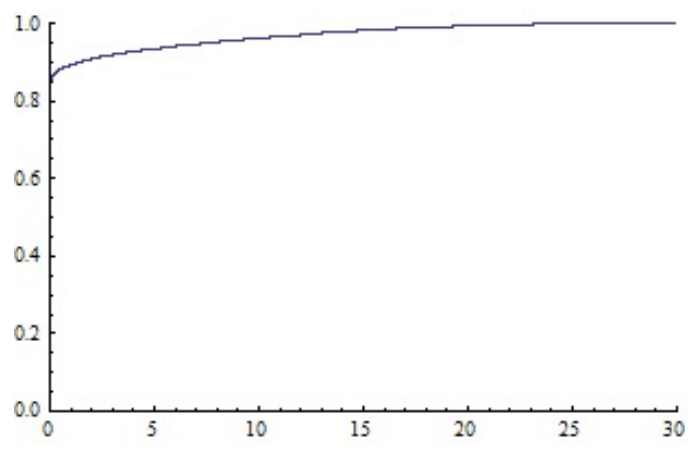

Figure 7: Cumulative distribution of all edges' "time to birth" in weeks. 


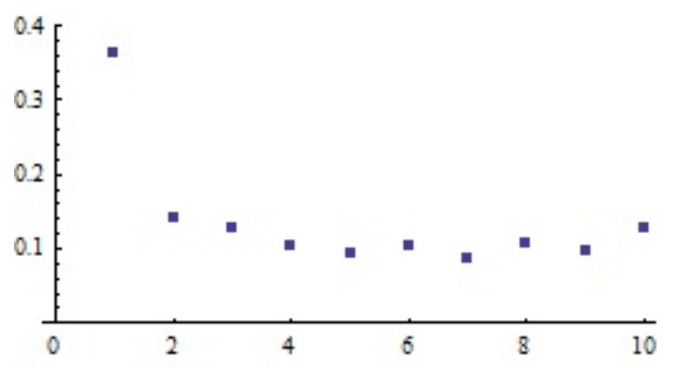

Figure 8: The fraction of edges unformed up to week $k-1$ and formed within week $k$, against $\mathrm{k}$ (estimates for $\alpha(k)$ ).

within the next time period (week).

Below we show the probability that such an edge is formed within the $k$ th week, given that it has not been formed in the first $(k-1)$ previous weeks, since both members had joined the network. This quantity is proportional to the weekly birth rate $\alpha(k)$. After the first week the probability that such an edge is born is 0.36 , the probability then settles approximately to 0.12 each week, as seen in Figure 8. There is thus evidence that the weekly birth rate is memory dependent, for the first few weeks at least, and is a decreasing function of the link's absence.

\section{Discussion}

The recent literature contains many attempts to describe evolving social network data that is observed from real communication systems (for example $[3,14])$. The availability of digital data from phone $[4,11]$, emails and social networking services and sites [10], means that such data descriptions are possible for very large data sets. It is useful to remark that a very similar descriptive phase of research took place, following the expositions of scale free [2] and small world [15] networks, which was largely concerned with static networks (work on proteomes, for example) and explanations about how such network structure could come about, using the preferential attachment mechanism, for example.

The recognition of the digital society and digital economy as providers of 
new spaces in which people can interact, entertain, and trade; coupled with the increasing need to make such activities secure, whilst recognising both aberrations and trends, means that data description, or extracting descriptive parameters is not enough. We need to define and test models that can be used to make inferences and forecasts about such evolving behaviour. Indeed the inverse problem of taking data (including errors) and estimating how it might correspond or not to alternative models is productive both in implying the nature of the underlying evolution at work [9], and in making predictions $[7,8]$.

In this paper we have focused on some simple models for evolving networks with memory. Long term memory effects may be important in that they introduce behaviour not observed in previous work on Markov models for evolving graphs [8]. Under such memory dependent assumptions we show that a range of long term behaviour is possible - from the full extinction, or full completion of the network, through heterogeneous quenched steady states, to constantly evolving dynamical equlibria. Moreover we have shown how, given a limited sample of observations, one might infer which of these eventualities is likely to occur, by estimating parameters, and examining the convergence of certain corresponding infinite products. Although such conclusions are caveatted by the assumptions of the model, they may well be actionable. For example, an awareness that the continuation of the current observed behaviour is enough to imply the eventual extinction or immortality of all possible connections, may lead to actions on the part of the system or service provider.

In future work we will examine the evidence for medium to long term memory dependence within a wider range of evolving communication networks.

We thank Haibo $\mathrm{Hu}$ for making the Wealink data available to us and we acknowledge the support of the EPSRC.

\section{References}

[1] Abramowitz, M. and Stegun, I. A. (Eds.). Handbook of Mathematical Functions with Formulas, Graphs, and Mathematical Tables, 9th printing. New York: Dover, 1972. 
[2] Barabasi, A.-L., Albert, R., Science, 286: 509-512, 1995.

[3] Brognat, P., Guillaume, Magnien, C., Robardet, C., Scherrer, A. Evolving Networks, preprint,

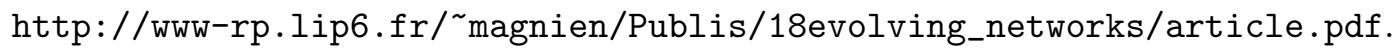

[4] Eagle, N., Pentland, A. and Lazer, D. Inferring social network structure using mobile phone data, Proceedings of the National Academy of Sciences, 106(36), pp. 15274-15278, 2009.

[5] Eun, Do Young. Theory of Evolving Networks. North Carolina State University. Spring 2009.

[6] Grindrod, P. Personal Communication, http://www.personal.reading.ac.uk/ sms06gp/TelCoTab.xls , 2010.

[7] Grindrod, P. Range dependent random graphs and their application to modelling large small world data sets, Physics Reviews E, 66, 2002.

[8] Grindrod, P., Higham, D. J. Dynamical Models for Evolving Networks. University of Reading, Proceedings of the Royal Society A, 466(2115), 753-770, 2010.

[9] Grindrod, P., Higham, D. J., Kalna, G. Periodic reordering, IMA $J$ of Num Analysis, 30(1), 195-207, 2010.

[10] Hu, Haibo., Wanga, Xiaofan. Evolution of a large online social network, Physics Letters A, Volume 373, Issues 12-13, Pages 1105-1110, 2009.

[11] Onnela, J.-P., Saramki, J., Hyvnen, J., Szab, G., Lazer, D., Kaski, K., Kertsz, J. and Barabasi, A.-L. Structure and tie strengths in mobile communication networks, PNAS May 1, vol. 104 no. 18 7332-7336, 2007.

[12] Rocha, L. E. C. Structural Evolution of the Brazilian Airport Network. Umea University. March 2009.

[13] Struble, R. A. Infinite Products Rescued: Chapter 1, Decreasing Products. North Carolina State University. July 2009. 
[14] Tang, J., Scellato, S., Musolesi, M., Mascolo, C., Latora, V. Small-world behavior in time-varying graphs. Cambridge, University of Cambridge, Universit'a di Catania, Scuola Superiore di Catania. September 2009.

[15] Watts, D.J. and Strogatz, S.H. Collective dynamics of 'small-world' networks, Nature 393 (6684): 40910, 1998.

[16] Weisstein, Eric W. "Raabe's Test." From MathWorld - A Wolfram Web Resource. http://mathworld.wolfram.com/RaabesTest.html

[17] Weisstein,

Eric

W.

"Pareto

Distribution." From MathWorld-A Wolfram Web Resource. http://mathworld.wolfram.com/ParetoDistribution.html

\section{Appendix}

We introduce a new quantity, $P_{i m m}^{*}(z), z=1,2, \ldots$ defined as the probability that an edge that has been present for exactly $z$ time steps, will eventually become immortal, irrespective of how many times it first dies and is then reborn prior to achieving immortality. Similarly we define $P_{i m m}^{*}(-z), z=$ $1,2, \ldots$ to be the probability that an edge that has been absent for exactly $z$ time steps, eventually becomes immortal, irrespective of how many times it dies and is reborn prior to achieving immortality.

Let us denote $P_{i m m}^{*}(1)=P_{i m m}^{*}$, then we can calculate $P_{i m m}^{*}$ in two ways: if we visualise a probability tree diagram, we can see that

$P_{i m m}^{*}=P_{i m m}+\left(1-P_{i m m}\right)\left(1-P_{e x t}\right) P_{i m m}+\left(\left(1-P_{i m m}\right)\left(1-P_{e x t}\right)\right)^{2} P_{i m m}+\ldots$

which is a simple geometric progression, and can easily be summed; or we can argue recursively, that after a single instant of death and rebirth our edge again has probability $P_{i m m}^{*}$ of eventually attaining immortality, hence

$$
P_{i m m}^{*}=P_{i m m}+\left(1-P_{i m m}\right)\left(1-P_{\text {ext }}\right) P_{i m m}^{*}
$$

In either case we obtain the result:

$$
P_{i m m}^{*}=\frac{P_{i m m}}{1-\left(1-P_{i m m}\right)\left(1-P_{e x t}\right)} .
$$


If we again visualise a probability tree diagram, then for $z=2,3, \ldots$, we see that the following must hold:

$$
P_{i m m}^{*}(z)=P_{i m m}(z)+\left(1-P_{i m m}(z)\right)\left(1-P_{e x t}\right) P_{i m m}^{*}
$$

and also that for $z=-1,-2, \ldots$ :

$$
P_{i m m}^{*}(z)=\left(1-P_{e x t}(z)\right) P_{i m m}^{*}
$$

It then follows that the analogously defined $P_{e x t}^{*}(z)$ satisfies

$$
P_{\text {ext }}^{*}(z)=1-P_{i m m}^{*}(-z)
$$

Equations (12-15) above allow us to calculate the probabilities of an edge eventually becoming immortal, or extinct, at any time step, covering all possible values of $z(= \pm 1, \pm 2, \ldots)$. 